\title{
Análisis económico del trastuzumab en Colombia
}

\author{
Economic analysis of trastuzumab in Colombia
}

\author{
- Hernán Carranza ${ }^{1,2}$, Carlos Vargas ${ }^{1,2}$, Laura Bernal ${ }^{1}$, Jorge Miguel Otero ${ }^{1,2}$, Andrés Felipe Cardona $a^{1,2}$ \\ 'Grupo Oncología Clínica y Traslacional, Instituto de Oncología, Fundación Santa Fe de Bogotá (Bogotá, Colombia). \\ ${ }^{2}$ Fundación para la Investigación Clínica y Molecular Aplicada del Cáncer (FICMAC); investigador asociado ONCOLGroup.
}

En abril de este año se publicó en Biomédica, el órgano de difusión del Instituto Nacional de Salud, una evaluación económica del trastuzumab como terapia adyuvante para el cáncer de seno $\mathrm{HER}_{2}$ positivo en Colombia; dicho análisis fue realizado por el Departamento de Farmacología y Toxicología de la Universidad de Antioquia (Medellín, Colombia) en colaboración con otros departamentos de salud pública a nivel regional. La conclusión sugiere que la administración del trastuzumab durante 12 meses no es una intervención costo-efectiva en nuestro país, afirmación que podría tener repercusiones significativas en la atención de las mujeres con cáncer de seno, en especial, si se considera que la utilización del monoclonal constituye una recomendación IA, basada en el mejor nivel de evidencia.

De igual forma, múltiples análisis de costo-efectividad llevados a cabo en otros países de referencia diseñados bajo el modelo de evaluación del tercer pagador favorecen el uso regular del trastuzumab en pacientes con enfermedad temprana y avanzada'. Al tratarse de un medicamento con gran impacto sobre los desenlaces primarios que ya se encuentra incluido en el Plan Obligatorio de Salud (POS) del Sistema General de Atención en Salud (SGAS), la información presentada por Buendía y colaboradores debe evaluarse con extrema rigurosidad ${ }^{2}$.

La validez externa del estudio es controvertible, dado que empleó la perspectiva del tercer pagador cuando el trastuzumab ya está incluido en el POS. Desde el 2010, se han presentado cambios sustanciales en el precio, en la tasa de cambio y en la incorporación de nuevas tecnologías en el sistema sanitario nacional; no obstante, el estudio no hizo referencia al proceso de actualización del POS, ejecutado por el Ministerio de la Protección Social en ese año y que avaló la incorporación al Plan de beneficios del trastuzumab. Esta decisión se basó en un reporte de la Universidad Nacional, en el cual, tras una descripción de la tecnología, una revisión sistemática de la literatura y una interpretación de los resultados con expertos locales, se recomendó la inclusión del trastuzumab y su utilización sin restricciones para los pacientes con cáncer de seno $\mathrm{HER}_{2}$ positivo ${ }^{3}$. Lo anterior basado en el impacto presupuestal, herramienta idónea para mostrar si un sistema de salud tiene la capacidad para financiar una nueva tecnología.

El mismo grupo mostró que el trastuzumab como terapia adyuvante en cáncer de seno temprano que sobreexpresa el oncogén $\mathrm{HER}_{2}$ tiene un impacto en la UPC del régimen subsidiado del 0,859\% y en la del contributivo del 0,707\%. Del mismo modo, en cáncer de seno metastásico, el impacto sobre la UPC del contributivo fue del $0,86 \%$ y del $0,707 \%$ en la del subsidiado. En el contexto actual, cualquier evaluación económica debería agregar valor en términos del impacto económico de la incorporación de dicha tecnología en el POS, lo que se omite en el texto en cuestión.

Por otro lado, es importante notar que, en la metodología descrita por Buendía y colaboradores, los costos y las utilidades fueron estimados a partir de la literatura, tomándolos de estudios anteriores y ajustándolos por la inflación actual a pesos colombianos. Por lo tanto, que los costos reportados sean extrapolados constituye un desliz metodológico de fuerza en una evaluación económica como esta, puesto que los costos son distintos en todos los contextos, evento por el que debieron utilizarse costos reales y actualizados para el caso local ${ }^{4}$. Las recomendaciones sobre la metodología reportadas en la literatura y la experiencia internacional establecen la existencia de factores que hacen que los datos económicos no sean fácilmente transferibles; por ejemplo, factores demográficos y epidemiológicos de la enfermedad, con amplias diferencias entre los distintos 
países por la estructura de edad de su población y la gran variabilidad en la incidencia y prevalencia de las enfermedades.

La disponibilidad de recursos asistenciales es otro elemento que no es extrapolable, debido a que la gama de tratamientos y las instalaciones sanitarias disponibles en cada entorno difieren significativamente. También existen variaciones en la práctica clínica entre regiones que cuentan con las mismas opciones terapéuticas, que, igualmente, pueden hacer variar el análisis de costoefectividad relativo de cada intervención. A su vez, los incentivos de remuneración en cada país afectan los precios o costos relativos. Así mismo, el emplear valores de utilidad estimados para otros países puede generar inconvenientes para interpretar los resultados en el ámbito colombiano, en especial, porque en el país no existen estimados sobre la utilidad.

Varios autores insisten en la relevancia del uso de datos locales y actuales en las evaluaciones económicas, y en la necesidad de indicar los costos unitarios por separado para aumentar la transparencia del análisis $5^{5,6}$. Aunque en todos los casos es fundamental reportar las fuentes de los datos y los cálculos efectuados para llegar a la información que alimenta el modelo empleado, este proceso de costos incluidos para la evaluación económica no es descrito con amplitud ni en la metodología ni en las tablas de datos del trabajo de Buendía y colaboradores.

En general, estos cálculos deben realizarse de forma estándar: el costo de un recurso es el producto de dos elementos, la cantidad total de recurso consumido y el valor monetario de la unidad de dicho recurso (por ejemplo, el costo de una hora de trabajo del profesional de salud, o el costo de un día de estancia hospitalaria). De ahí, el análisis de costos requiere de tres procesos definidos: 1) identificar los recursos: ¿qué uso de los recursos es probable en un programa o tratamiento?, este punto se refiere a listar los recursos por costear; 2 ) medir: ¿qué volumen de recursos podrían ser usados en un programa o tratamiento?; y 3) valorar: ¿cuál es el valor de los recursos utilizados??

El trastuzumab es un medicamento que tiene el precio regulado en Colombia por la Comisión Nacional de Precios de Medicamentos y Dispositivos Médicos (CNPM-DM); en la actualidad, tiene un valor máximo de recobro registrado en la Circular 4 del 8 noviembre de 2012, publicada en el Diario Oficial № 48608; en el estudio no hay claridad sobre el uso de esta fuente. Adicionalmente, tampoco se describe el valor del PIB per capita empleado para el análisis, únicamente se hizo referencia dentro de la bibliografía a una consulta en la página oficial del Banco Mundial para el 2012, mientras que en el documento se referencia un ajuste por inflación al 2010. Por todo lo anterior, la metodología genera algunas dudas sobre los resultados del modelo para nuestro país, restándole relevancia. Son muchas las limitaciones de basar nuestra evaluación económica en los resultados de las consideraciones hechas en otros lugares, motivo que hace necesario reconsiderarlos y ajustarlos de forma meticulosa antes de extrapolarlos 8 .10.

En cuanto a la conclusión, el único criterio que usa el estudio para afirmar que el trastuzumab como tratamiento adyuvante en cáncer de mama $\mathrm{HER}_{2}$ positivo no es costo-efectivo en Colombia es la definición del umbral propuesto por la Organización Mundial de la Salud (OMS) que contempla tres veces el PIB per capita. Sin embargo, al realizar evaluaciones económicas para medicamentos empleados en fases terminales, es necesario considerar elementos de peso que van más allá del costo, ya que en este tipo de escenarios no es aceptable rechazar los tratamientos que demuestran ser capaces de extender la vida solo con criterios de costo-efectividad.

Así lo argumenta el National Institute for Health and Care Excellence (NICE), al admitir que en estos pacientes es apropiado financiar el uso de tratamientos costo-efectivos que se encuentren por encima del umbral de referencia ${ }^{11}$. Incluso, es válido considerar la construcción del umbral a partir del gasto que ya se está asumiendo (terapia tradicional), puesto que, en este tipo de enfermedades, la atención del umbral propuesta por el Banco Interamericano de Desarrollo (BID) y la OMS (de 1 y hasta 3 veces el PIB per capita) será pequeña. Para tomar estas decisiones, algunos países han comenzado a definir nuevos umbrales de costo-efectividad; conceptualmente hablando, se trata de precisar qué tantos recursos adicionales está dispuesta a invertir una sociedad en salud y calidad de vida agregados, cantidad que suele definirse utilizando estándares de precios en dólares ${ }^{12}$. El costo de la terapia sin trastuzumab en el estudio publicado es de 75.315 dólares (144.898.528 pesos colombianos), lo que tampoco clasificaría dentro de la disponibilidad por pagar tomando como referencia el umbral citado. 
La literatura mundial es enfática en los beneficios y la costo-efectividad de este tratamiento. Un estudio publicado en los Estados Unidos en el 2010 estimó, a partir de un modelo de Monte Carlo, que el uso de trastuzumab adyuvante podía prevenir un 38\% de las recurrencias en mujeres $\mathrm{HER}_{2}$ positivas, con una reducción total en los costos directos e indirectos que osciló entre 240 millones y 1,7 billones de dólares ${ }^{13}$. En el 2012, estos resultados fueron reiterados en un estudio publicado en Canadá, que evaluó una ganancia de 1,38 años de vida ajustados por calidad (QALY) y de 1,17 años de vida ganados con una relación costoefectividad satisfactoria. Así mismo, en Europa se ha realizado otra serie de evaluaciones de costo-efectividad con resultados positivos en diversos países ${ }^{14-16}$.

En el Reino Unido, se reportó el análisis de 239 pacientes en los que se observó una mejoría significativa de la supervivencia global (SG) a 3 años en el grupo tratado con trastuzumab $(98,5$ vs. $87,6 \%)$, al igual que en la supervivencia libre de recaída (SLR) (90,3 vs. $73,3 \%)^{17}$. Otro trabajo efectuado en Suiza mostró un impacto positivo en la SG a 10 y 15 años (81,8 vs. 66,1\% y 73,6 vs. $57,0 \%$, respectivamente) junto con un ahorro de 8.497 euros por cada mujer después de 10 años de tratamiento con trastuzumab, y de 9.256 euros a los 15 años. En Asia, la costo-efectividad del trastuzumab se estableció en Japón ${ }^{18}$ y en China ${ }^{19}$, donde se encontró que la utilización del monoclonal en adyuvancia para el cáncer de seno temprano puede prolongar la vida en 2,87 años, en comparación con la quimioterapia convencional, hallazgo que fue costo-efectivo.

Esta información favorece a la prudencia e invita a la reflexión sobre la conducción e interpretación de los estudios de economía en salud en cáncer hechos en Colombia.

\section{Referencias}

1. Carlson RW, Moench SJ, Hammond ME, Perez EA, Burstein HJ, et al.; NCCN HER2 Testing in Breast Cancer Task Force. HER2 testing in breast cancer: NCCN Task Force report and recommendations. J Natl Compr Canc Netw. 2006;4 Suppl 3:S1-22; quiz S23-4.

2. Buendía JA, Vallejos C, Pichón-Rivière A. Evaluación económica del trastuzumab como tratamiento adyuvante en cáncer de mama HER2-positivo en Colombia. Biomédica. 2013;33(3).

3. Comisión de Regulación en Salud. Reporte de evaluación de posible inclusión al POS para trastuzumab. Universidad Nacional de Colombia, Facultad de Medicina, Instituto de Investigaciones Clínicas.

4. Ministerio de Salud y Protección Social, Colciencias, Centro de Estudios e Investigación en Salud de la Fundación Santa Fe de Bogotá, Escuela de Salud Pública de la Universidad de Harvard. Guía metodológica para el desarrollo de guías de atención integral.
5. Sculpher MJ, Pang FS, Manca A, Drummond MF, Golder S, Urdahl $\mathrm{H}$, et al. Generalisability in economic evaluation studies in healthcare: a review and case studies. Health Technol Assess. 2004;8(49):1-192.

6. Drummond M, Barbieri M, Cook J, Glick HA, Lis J, Malik F, et al. Transferability of economic evaluations across jurisdictions: ISPOR Good Research Practices Task Force report. Value Health. 2009;12(4):409-18

7. Drummond MF, Sculper MJ, Torrance GW, O'Brien BJ, Stoddart $\mathrm{GL}$. Methods for the economic evaluation of health care programmes. Oxford: Oxford University Press; 2005.

8. Chicaíza L, Vega R. Determinantes de la utilización de la evaluación económica en la toma de decisiones dentro del sistema de salud colombiano. Innovar Revista de Ciencias Administrativas y Sociales, Universidad Nacional de Colombia. 2012;18(31):77-9.

9. Garau M, Shah KK, Mason AR, Wang Q, Towse A, Drummond MF. Using QALYs in cancer: a review of the methodological limitations. Pharmacoeconomics. 2011:29(8):673-85.

10. Ferrusi IL, Leighl NB, Kulin NA, Marshall DA. Do economic evaluations of targeted therapy provide support for decision makers? ajmc.com [online] 2011 [cited 2013 Jul 8]. Available from: <http://www.ajmc.com/publications/supplement/2011/ AJMC_11JOPmay/AJMC_11JOPmay_Ferrusi_SP61 to70/4>

11. NICE. Appraising life-extending, end of life treatments. 2009. Avalilable from: <http://www. nice.org.uk/media/88A/F/ supplementaryAdviceTACEoL.pdf>

12. BID. Método de la costo-efectividad incremental. 2013. Claxton K, Martin S, Soares M, Rice N, Spackman E, Hinde S, et al. Methods for the estimation of the NICE cost effectiveness threshold.

13. Danese MD, Lalla D, Brammer M, Doan Q, Knopf K. Estimating recurrences prevented from using trastuzumab in HER-2/neupositive adjuvant breast cancer in the United States. Cancer. 2010;116(24):5575-83

14. National Institute for Health and Clinical Excellence. Trastuzumab for the adjuvant treatment of early-stage HER2-positive breast cancer. NICE technology appraisal guidance 107, 2006.

15. Dedes KJ, Szucs TD, Imesch P, Fedier A, Fehr MK, Fink D. Costeffectiveness of trastuzumab in the adjuvant treatment of early breast cancer: a model-based analysis of the HERA and FinHer trial. Ann Oncol. 2007;18(9):1493-9.

16. Purmonen TT, Pänkäläinen $E$, Turunen $J H$, Asseburg $C$, Martikainen JA. Short-course adjuvant trastuzumab therapy in early stage breast cancer in Finland: cost-effectiveness and value of information analysis based on the 5-year follow-up results of the FinHer Trial. Acta Oncol. 2011;50(3):344-52.

17. Webster RM, Abraham J, Palaniappan N, Caley A, Jasani B, Barrett-Lee P. Exploring the use and impact of adjuvant trastuzumab for HER2-positive breast cancer patients in a large UK cancer network. Do the results of international clinical trials translate into a similar benefit for patients in South East Wales? Br J Cancer. 2012;106(1):32-8.

18. Shiroiwa T, Fukuda T, Shimozuma K, Ohashi $Y$, Tsutani K. The model-based cost-effectiveness analysis of 1 -year adjuvant trastuzumab treatment: based on 2-year follow-up HERA trial data. Breast Cancer Res Treat. 2008;109(3):559-66.

19. Chen W, Jiang Z, Shao Z, Sun Q, Shen K. An economic evaluation of adjuvant trastuzumab therapy in HER2-positive early breast cancer. Value Health. 2009;12 Suppl 3:S82-4. 